\title{
Unmasking the amphotericin $B$ resistance mechanisms in Candida haemulonii species complex
}

\author{
Laura N. Silva ${ }^{1}$, Simone S. C. Oliveira ${ }^{1}$, Lucas B. Magalhães ${ }^{1}$, Valter V. \\ Andrade Neto' ${ }^{2}$ Eduardo C. Torres-Santos ${ }^{2}$, Mariana D. C. Carvalho ${ }^{3}$, \\ Marcos D. Pereira ${ }^{3}$, Marta H. Branquinha ${ }^{1}$ and André L. S. Santos ${ }^{1,4, *}$
}

${ }^{1}$ Laboratório de Estudos Avançados de Microrganismos Emergentes e Resistentes, Departamento de Microbiologia Geral, Instituto de Microbiologia Paulo de Góes - Bloco E subsolo sala 05- Universidade Federal do Rio de Janeiro, Rio de Janeiro, 21941-902, Brazil; ${ }^{2}$ Laboratório de Bioquímica de Tripanosomatídeos, Instituto Oswaldo Cruz, Av. Brasil, 4365 - Fundação Oswaldo Cruz, Rio de Janeiro, 21040-360, Brazil;

${ }^{3}$ Laboratório de Citotoxicidade e Genotoxicidade, Instituto de Química, Centro de Tecnologia, Av. Athos da Silveira Ramos, 149 - bloco A, $5^{\circ}$ andar - Universidade Federal do Rio de Janeiro, Rio de Janeiro, 21941-909, Brazil;

${ }^{4}$ Programa de Pós-Graduação em Bioquímica, Instituto de Química, Centro de Tecnologia, Av. Athos da Silveira Ramos, 149 - bloco A, $5^{\circ}$ andar - Universidade Federal do Rio de Janeiro, Rio de Janeiro, 21941-909, Brazil;

* Corresponding author.

E-mail address: andre@micro.ufrj.br 
Table S1.: Antifungal susceptibility profiles of $C$. haemulonii complex species using CLSI method.

\begin{tabular}{|c|c|c|c|c|c|}
\hline \multirow[t]{2}{*}{ Candida species (codes) } & \multicolumn{5}{|c|}{$\operatorname{MIC}(\mathrm{mg} / \mathrm{L})^{\mathrm{a}}$} \\
\hline & AMB & FLC & ITC & VRC & CAS \\
\hline C. duobushaemulonii (LIPCh6) & $16(\mathrm{R})$ & $>64(\mathrm{R})$ & 0.5 (SDD) & $1(\mathrm{~S})$ & $0.25(\mathrm{~S})$ \\
\hline C. duobushaemulonii (LIPCh9) & $2(\mathrm{R})$ & $>64(\mathrm{R})$ & $8(\mathrm{R})$ & $>16(\mathrm{R})$ & $0.25(\mathrm{~S})$ \\
\hline C. haemulonii (LIPCh4) & $4(\mathrm{R})$ & $>64(\mathrm{R})$ & $>16(\mathrm{R})$ & $>16(\mathrm{R})$ & $0.5(\mathrm{~S})$ \\
\hline C. haemulonii (LIPCh7) & $2(\mathrm{R})$ & $>64(\mathrm{R})$ & $6(\mathrm{R})$ & $6(\mathrm{R})$ & $0.25(\mathrm{~S})$ \\
\hline C. haemulonii var. vulnera (LIPCh5) & $2(\mathrm{R})$ & $>64(\mathrm{R})$ & $0.5(\mathrm{SDD})$ & $0.5(\mathrm{~S})$ & $0.25(\mathrm{~S})$ \\
\hline C. haemulonii var. vulnera (LIPCh8) & $8(\mathrm{R})$ & $>64(\mathrm{R})$ & $8(\mathrm{R})$ & $>16(\mathrm{R})$ & $0.125(\mathrm{~S})$ \\
\hline C. krusei (ATCC 6258) & $0.25(\mathrm{~S})$ & $64(\mathrm{R})$ & $1(\mathrm{R})$ & $0.5(\mathrm{~S})$ & $0.03(\mathrm{~S})$ \\
\hline C. lusitaniae (ATCC 200950) & $1(\mathrm{R})$ & $1(\mathrm{~S})$ & $0.06(\mathrm{~S})$ & $0.06(\mathrm{~S})$ & $0.03(\mathrm{~S})$ \\
\hline C. tropicalis (ATCC 750) & $0.25(\mathrm{~S})$ & $0.5(\mathrm{~S})$ & $0.03(\mathrm{~S})$ & $0.03(\mathrm{~S})$ & $0.03(\mathrm{~S})$ \\
\hline
\end{tabular}

a MIC, minimal inhibitory concentration; AMB, amphotericin B; FLC, fluconazole; ITC, itraconazole, VRC, voriconazole; CAS, caspofungin; R, resistant; S, susceptible. The clinical breakpoints defined for Candida tropicalis was used for the interpretation of MIC data as follows: susceptible (S) $\leq 2 \mathrm{mg} / \mathrm{L}$, susceptible-dose dependent (S-DD) $4 \mathrm{mg} / \mathrm{L}$, resistant (R) $\geq 8 \mathrm{mg} / \mathrm{L}$ for fluconazole; $\mathrm{S} \leq 0.12 \mathrm{mg} / \mathrm{L}, \mathrm{S}-\mathrm{DD} 0.25-0.5 \mathrm{mg} / \mathrm{L}, \mathrm{R} \geq 1 \mathrm{mg} / \mathrm{L}$ for voriconazole; and $\mathrm{S} \leq 0.25 \mathrm{mg} / \mathrm{L}, \mathrm{S}-\mathrm{DD} 0.5 \mathrm{mg} / \mathrm{L}, \mathrm{R} \geq 1 \mathrm{mg} / \mathrm{L}$ for caspofungin. The clinical breakpoints defined for Candida krusei was: susceptible (R) intrisically resistant to fluconazole; $\mathrm{S} \leq 0.5$ $\mathrm{mg} / \mathrm{L}, \mathrm{S}-\mathrm{DD} 1 \mathrm{mg} / \mathrm{L}, \mathrm{R} \geq 2 \mathrm{mg} / \mathrm{L}$ for voriconazole; and $\mathrm{S} \leq 0.25 \mathrm{mg} / \mathrm{L}, \mathrm{I} 0.5 \mathrm{mg} / \mathrm{L}, \mathrm{R} \geq 1$ $\mathrm{mg} / \mathrm{L}$ for caspofungin. *The CLSI has not defined interpretative cutoffs for AMB and ITR. In general, Candida isolates with MIC $>1 \mathrm{mg} / \mathrm{L}$ are likely to be resistant to AMB and ITR. 
Table S2.: Drug susceptibility profiles of C. haemulonii strains using mitochondrial respiratory chain inhibitors and synergism with AMB (mg/L).

\begin{tabular}{|c|c|c|c|c|c|c|}
\hline Combination & $\begin{array}{l}\text { MIC } \\
\text { alone }\end{array}$ & $\begin{array}{c}\text { MIC } \\
\text { combined* }\end{array}$ & $\begin{array}{l}\text { MIC } \\
\text { alone }\end{array}$ & $\begin{array}{c}\text { MIC } \\
\text { combined* }\end{array}$ & $\begin{array}{l}\text { MIC } \\
\text { alone }\end{array}$ & $\begin{array}{c}\text { MIC } \\
\text { combined* }\end{array}$ \\
\hline Strains & Ch4 & & Ch8 & & & \\
\hline AMB & 4 & & 8 & & 2 & \\
\hline Rotenone (Complex I) mM & $>0.3$ & & $>0.3$ & & $>0.3$ & \\
\hline AMB + Rotenone (Complex I) & & 4 & & 8 & & 2 \\
\hline AMB & 4 & & 8 & & 2 & \\
\hline Carboxine (Complex II) mM & $>0.2$ & & $>0.2$ & & $>0.2$ & \\
\hline AMB + Carboxine (Complex II) & & 4 & & 8 & & 2 \\
\hline AMB & 4 & & 8 & & 2 & \\
\hline Antimycin A (Complex III) mM & $>0.003$ & & $>0.003$ & & $>0.003$ & \\
\hline AMB + Antimycin A (Complex III) & & 1 & & 2 & & 0,5 \\
\hline $\mathrm{AMB}$ & 4 & & 8 & & 2 & \\
\hline Potassium cyanide (Complex IV) mM & $>0.01$ & & $>0.01$ & & $>0.01$ & \\
\hline AMB + Potassium cyanide (Complex IV) & & 4 & & 8 & & 2 \\
\hline AMB & 4 & & 8 & & 2 & \\
\hline Salicylhydroxamic acid (Alternative oxidase AOX) $\mathrm{mM}$ & $>5$ & & $>5$ & & $>5$ & \\
\hline AMB + Salicylhydroxamic acid (Alternative oxidase AOX) & & $\mathbf{0 , 5}$ & & 1 & & 0,25 \\
\hline
\end{tabular}

* Minimal inhibitory concentration (MIC) of AMB in the presence of the highest concentration of mitochondrial respiratory chain inhibitors tested. 


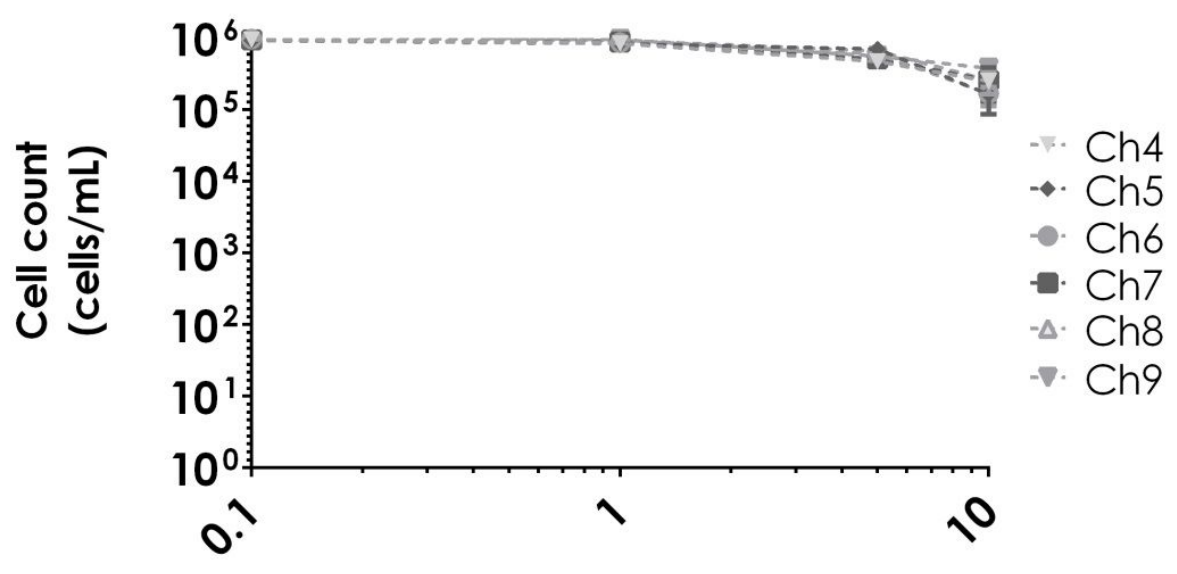

$\mathrm{AMB}(\mathrm{mg} / \mathrm{L})$

Figure S1. Killing effect of AMB on C. haemulonii strains. Fungal isolates were incubated with different concentrations of $\mathrm{AMB}$ for $3 \mathrm{~h}$ and then spread in a solid Sabouraud medium in order to count the number of CFUs after $48 \mathrm{~h}$. 